\title{
Low Grade Astrocytoma in the Medial Part of the Frontal Lobe Manifesting as Paroxysmal Speech Disturbance \\ - Case Report-
}

\author{
Shingo TAKano, Tooru MARUNo, Yasushi SHIBATA, \\ Kiyoyuki YANAKA, and Shizuo SHIRAI
}

Department of Neurosurgery, Soujinkai Hospital, Ibaraki

\begin{abstract}
A right-handed 48-year-old female had clustered groups of seizures characterized by speech disturbance (attacks of verbal repetition and speech arrest) over a period of 7 years. Later, speech arrest was accompanied by perioral focal seizures. Magnetic resonance imaging and surgery found a low grade astrocytoma, centered in the left supplementary motor area. Postoperatively, no seizures or speech disturbances occurred for 3 years. This case confirms the main function of the supplementary motor area of the dominant hemisphere is connected with the initiation of speech and the setting into motion of the mechanism of speech.
\end{abstract}

Key words: astrocytoma, supplementary motor area, speech arrest

\section{Introduction}

The supplementary motor area (SMA) is located in the medial part of the left (dominant side) of the frontal lobe. The SMA has been considered a speech area separate from Broca's and Wernicke's areas since Penfield and Welch ${ }^{13)}$ demonstrated that electrical stimulation of the SMA caused speech arrest. The anatomical boundaries of the SMA, determined by stimulation mapping, are the leg motor representation posteriorly and the cingulate sulcus inferiorly. The lateral and anterior borders are less precise but probably do not extend beyond the superior frontal gyrus either laterally or more than $5 \mathrm{~cm}$ anteriorly. ${ }^{13,16)}$ However, disturbance of the SMA associated with intracranial tumors in humans is not well recognized.

We report a patient with low grade astrocytoma in the medial frontal lobe manifesting as speech arrest and vocal repetition.

\footnotetext{
Received May 30, 1995; Accepted September 6, 1995
}

\section{Case Report}

A 48-year-old female was admitted on July 2, 1992, for the evaluation of focal seizure in her face followed by speech disturbance. Her first seizure had occurred 7 years previously. The seizure started with sudden vocal repetition for a few minutes followed by unconsciousness. She regained normal consciousness rapidly and there were no speech disturbances or neurological deficits after the seizure. Computed tomography $(\mathrm{CT})$ at that time revealed a faint low density area in the frontal lobe with no postcontrast enhancement (Fig. 1). Her seizures were controlled by anticonvulsants, but continued to occur once a month with irregular medication. Two weeks before admission, the frequency of seizures increased. The seizures started with abnormal oral movement followed by speech arrest for few minutes, during which she understood spoken commands but could not write. There was no paresis, neurological signs or symptoms except for the seizure.

Magnetic resonance (MR) imaging demonstrated an irregular shape mass with minimal perifocal ede$\mathrm{ma}$ in the medial side of the left frontal lobe (Fig. 2A, B). Sagittal and coronal images showed this le- 


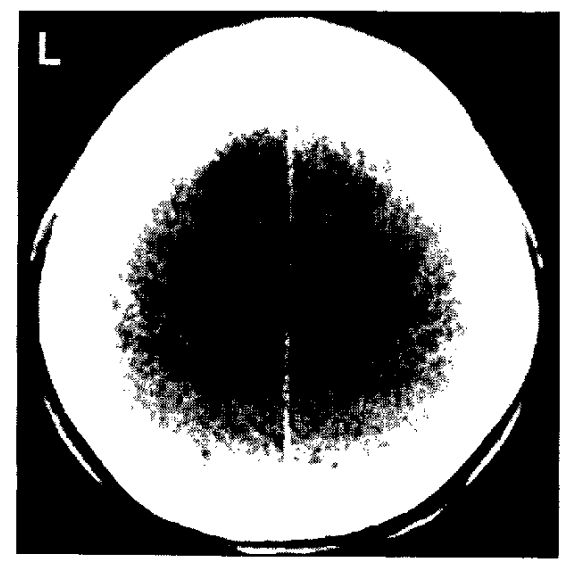

Fig. 1 Postcontrast CT scan 7 years before admission demonstrating a faint low density area in the left frontal lobe.

sion was located in the SMA, limited by the leg motor representation posteriorly, the cingulate sulcus inferiorly, and did not extend beyond the superior frontal gyrus either laterally or by more than $5 \mathrm{~cm}$ anteriorly (Fig. 2C, D). MR imaging with gadoliniumdiethylenetriaminepenta-acetic acid (Gd-DTPA) showed no abnormal enhancement. Carotid angiography showed no tumor staining. The interictal electroencephalography did not identify paroxysmal activity.

A left frontal craniotomy was performed, which revealed the intact cortical surface of the medial part of the frontal lobe. A subcortical tumor was observed and nearly totally removed. The histological diagnosis was low grade astrocytoma (Fig. 3). Whole brain irradiation was performed with a total dose of $60 \mathrm{~Gy}$.

The postoperative and postradiation courses were uneventful. No seizures or tumor recurrence have been observed for 3 years after the operation (Fig. 4).

\section{Discussion}

Our case illustrates the important role of the SMA in regulating the speech function, based on the clear MR imaging localization of the lesion in the SMA, the paroxysmal speech disturbances as the only symptom, and the disappearance of the symptoms following removal of the lesion. Speech modulation is caused by stimulation, or seizures located in the SMA. Regional cerebral blood flow data have shown simultaneous activation of the bilateral SMAs during speech, with a slight predominance of flow on the left. ${ }^{9)}$ Laplane et $a .^{8)}$ described stereo-
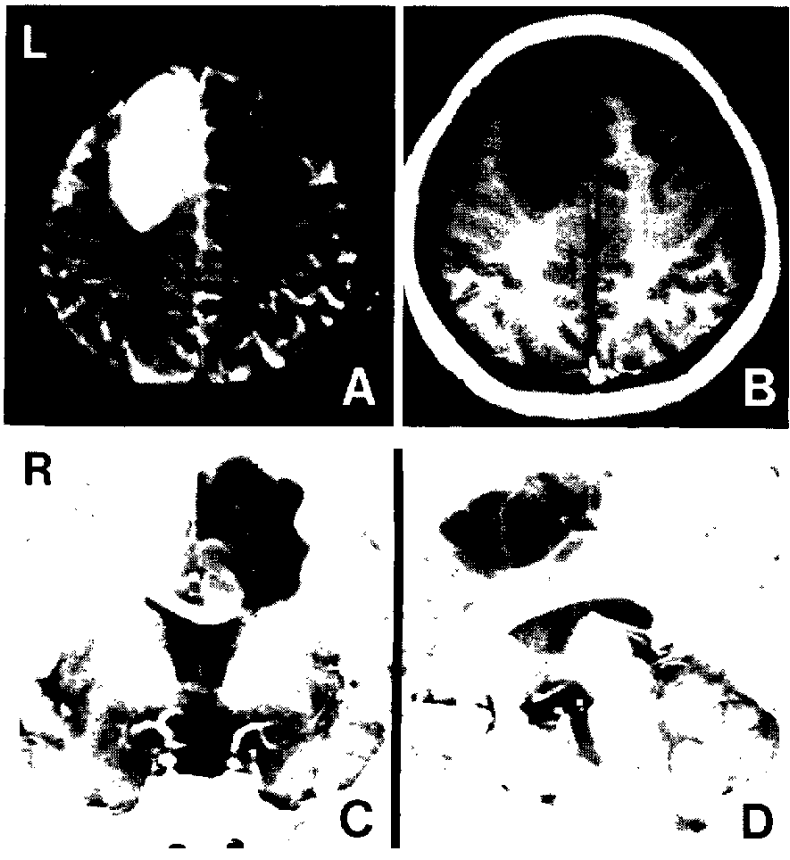

Fig. 2 Axial $T_{2}$-weighted MR image (A) on admission demonstrating a hyperintense irregular shape mass with minimal perifocal edema in the medial side of the left frontal lobe. Axial $T_{1}$-weighted MR image with Gd-DTPA (B) showing the hypointense lesion with no enhancement. Sagittal (C) and coronal (D) $\mathrm{T}_{2}$-weighted MR images demonstrating the lesion mainly located in the SMA. The lesion is limited by the leg motor representation posteriorly, the cingulate sulcus inferiorly, and does not extend beyond the superior frontal gyrus either laterally or by more than $5 \mathrm{~cm}$ anteriorly.

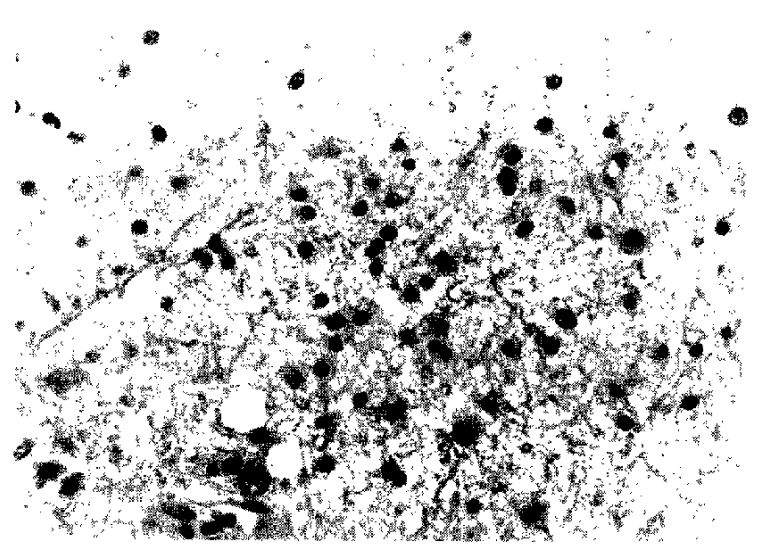

Fig. 3 Photomicrograph of surgical specimen showing features of low grade astrocytoma. HE stain, $\times 200$. 


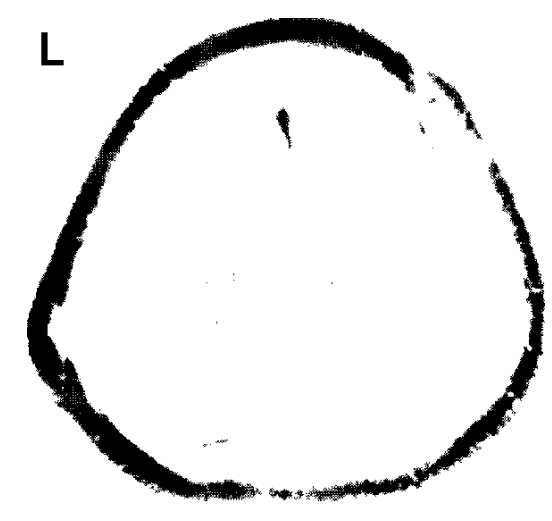

Fig. 4 Axial $\mathrm{T}_{1}$-weighted MR image with Gd-DTPA 3 years after the operation revealing no tumor recurrence.

tactic SMA resections of epileptogenic foci in three patients, resulting in global akinesis and speech arrest postoperatively. Recent electrical stimulation studies demonstrated the presence of somatotopic organization in the human SMA, with the lower extremities represented posteriorly, the head and face anteriorly, and the upper extremities between these two regions. Vocalization and speech arrest or slowing of speech were evoked anterior to the representation of the face..$^{5)}$ Rostomily et $a l .{ }^{14)}$ described five patients with primary glial tumors in the SMA who manifested speech disturbances, including hesitation and initiation difficulties in spontaneous speech, transient word-finding difficulty, and transient dysnomia. These disturbances were reversed postoperatively. Another electrical stimulation study showed negative motor response to stimulation of the rostral part of the SMA, which explains the mechanism of speech arrest due to SMA lesions. ${ }^{10)}$ These results indicate that the SMA is closely related to the speech function, especially the initiation of speech and the setting into motion of the mechanism of speech. ${ }^{1)}$

Anatomical studies have found connections between the SMA and all components of the motor system, including the bilateral motor cortices, cingulate gyri, contralateral SMA, and caudate and spinal cord with basal ganglia input via the thalamus. ${ }^{7,14,15,18)}$ Clinical observations also show important functions of the SMA other than speech modulation. Rostomily $e t a l .{ }^{14)}$ described the SMA syndrome consisting of reversible contralateral weakness, neglect, mutism, and impaired initiation dysfunction, such as paucity of spontaneous movement and hesitancy in activating volitional movement as well as apraxia, in patients following total or nearly total resection of primary and metastatic tumors involving the dominant hemisphere SMA. The SMA appears to be involved in the planning or initiation of motor activity. Persistent alien hand, which is characterized by unwilling and uncontrolled actions of the upper limb, is associated with an ischemic lesion localized in the SMA. ${ }^{17)}$ Hemiparkinsonism due to impairment of the basal ganglia output to the SMA has been demonstrated in a patient with a falx meningioma in the SMA. ${ }^{11)}$

Clinically, speech disturbances due to SMA lesions may be divided into paroxysmal and permanent types. Many patients with parasagittal and/or falx meningiomas develop permanent speech disturbances, such as lack of speech initiative, anomia, and expressive dysphasia, usually associated with motor weakness of the extremities. ${ }^{1,4)}$ Speech arrest is the paroxysmal speech disturbance most frequently encountered clinically in patients with space-occupying lesions of the SMA area. ${ }^{1)}$ The patient is unable to speak during the seizures, but preserves the faculty of understanding speech. Attacks of speech arrest are associated with verbal repetition and vocalization." Verbal repetition is the involuntary saying of the same words over and over again. A tumor in the SMA usually manifests as paroxysmal speech arrest associated with transient or permanent limb dysfunction. ${ }^{1,6)}$ Our case is an unusual example of epileptic arrest of speech without limb dysfunction. Such seizures may be followed by adversive seizures ${ }^{1-3,12)}$ or focal seizure of face and upper extremity. ${ }^{14)}$ Based on the somatotopic organization in the SMA, the causative lesion would be located in the rostral part of SMA, anterior to the supplementary motor representation of the face. ${ }^{5,10)}$ The speech arrest may occur in either the dominant or non-dominant SMA, confirmed by an electrical stimulation study in patients with intractable epilepsy. ${ }^{5)}$ All previous cases have involved a slowgrowing lesion; three low grade astrocytomas, one meningioma, one oligodendroglioma, and one mixed oligoastrocytoma. Paroxysmal speech arrest without limb dysfunction seems to be a clinical manifestation of such a localized SMA lesion. In our case, the faint low density area at the medial side of the high frontal lobe was hard to detect at the initial CT scan. Growth of the tumor resulted in a remarkable lesion in the SMA. Coronal and/or sagittal MR images provided precise anatomical location of the lesion in the SMA.

The present case confirmed the main function of the SMA of the dominant hemisphere is connected with the initiation of speech and the setting into mo- 
tion of the mechanism of speech. Speech arrest and verbal repetition are clinical manifestations of slow-growing tumors in the medial part of the frontal lobe.

\section{References}

1) Arseni C, Botez MI: Speech disturbances caused by tumours of the supplementary motor area. Acta Psychiatr Neurol Scand 36: 279-299, 1961

2) Botez M, Wertheim N: Expressive aphasia and amusia following right frontal lesion in a right handed man. Brain 82: 186-202, 1959

3) Caplan LR, Zervas NT: Speech arrest in a dextral with a right mesial frontal astrocytoma. Arch Neurol 35: 252-253, 1978

4) Chusid JG, de Guiterrez-Mahoney CG, MargulesLavergne MP: Speech disturbances in association with parasagittal frontal lesions. $J$ Neurosurg 11: 193-204, 1954

5) Fried I, Katz A, McCarthy G, Sass KJ, Williamson $P$, Spencer SS, Spencer DD: Functional organization of human supplementary motor cortex studied by electrical stimulation. $J$ Neurosci 11:3656-3666, 1991

6) Guidetti B: Désordes de la parole associes à des lésions de la surface interhémisphérique frontale postérieure. Rev Neurol (Paris) 97: 121-131, 1957

7) Jurgens $U$ : The efferent and afferent connections of the supplementary motor area. Brain Res 300: 63-81, 1984

8) Laplane $D$, Talairach J, Meininger V, Bancaud J, Orgogozo JM: Clinical consequences of corticectomies involving the supplementary motor area in man. J Neurol Sci 34: 301-314, 1977

9) Larsen B, Skinhoj E, Lassen NA: Variations in regional cortical blood flow in the right and left hemispheres during automatic speech. Brain 101: 193-209, 1978

10) Lim SH, Dinner DS, Pillay PK, Luders H, Morris
HH, Klem G, Wyllie E, Awad IA: Functional anatomy of the human supplementary sensorimotor area: Results of extraoperative electrical stimulation. Electroencephalogr Clin Neurophysiol 91: 179-193, 1994

11) Miyagi Y, Morioka T, Otsuka M, Fukui M: Striatal glucose metabolism and $\left[{ }^{18} \mathrm{~F}\right]$ Fluorodopa uptake in a patient with tumor-induced hemiparkinsonism. $\mathrm{Neu}$ rosurgery 32: 838-841, 1993

12) Peled R, Harnes B, Borovich B, Sharf B: Speech arrest and supplementary motor area seizures. Neurology 34: 110-111, 1984

13) Penfield $W$, Welch $K$ : The supplementary motor area of the cerebral cortex: A clinical and experimental study. Arch Neurol Psychiatry 66: 289-317, 1951

14) Rostomily RC, Berger MS, Ojemann GA, Lettich E: Postoperative deficits and functional recovery following removal of tumors involving the dominant hemisphere supplementary motor area. $J$ Neurosurg 75: 62-68, 1991

15) Schell GR, Strick PL: The origin of thalamic inputs to the arcuate premotor and supplementary motor areas. J Neurosci 4: 539-560, 1984

16) Talairach J, Bancaud J: The supplementary motor area in man (anatomo-functional findings by stereoelectro-encephalography in epilepsy). Int $J$ Neurol 5: 330-347, 1966

17) Trojano L, Crisci C, Lanzillo B, Elefante R, Caruso G: How many alien hand syndromes? Follow-up of a case. Neurology 43: 2710-2712, 1993

18) Wiesendanger $M$, Wiesendanger $R$ : The supplementary motor area in the light of recent investigations. Exp Brain Res Suppl 9: 382-392, 1984

Address reprint requests to: S. Takano, M.D., Department of Neurosurgery, Soujinkai Hospital, 1467 Oka, Fujishiro-machi, Kitasouma-gun, Ibaraki 300-15, Japan. 